\title{
Amélioration de la qualité de fragmentation des roches fissurées lors de l'abattage à l'explosif dans les mines à ciel ouvert
}

\author{
par Djoudi MERABET, Hamid KHERBACH \\ Université de Béjaia (Algérie), \\ Djamel MEHRI \\ Université d'Annaba (Algérie)
}

\section{Introduction}

L'un des maillons des processus technologiques de l'exploitation des gisements des minéraux à ciel ouvert est la préparation des roches à l'extraction. Cette étape de préparation prédétermine le rendement des engins, la sécurité du travail et, en général, l'efficacité des travaux à ciel ouvert. Dans la plupart des cas, la préparation des roches à l'extraction repose sur la destruction du massif des roches jusqu'à l'obtention de morceaux de dimensions nécessaires et admissibles pour le travail normal de tout le complexe d'extraction et de transport. Tout comme elle doit assurer un degré minimal de mélange de roches stériles et de mineral afin d'avoir la plus petite dilution possible. Le processus de l'abattage des roches à l'explosif occupe une place importante dans le système de gestion de la qualité des minerais extraits. L'amélioration de la qualité de la fragmentation peut être obtenue en agissant sur plusieurs facteurs parmi lesquels les plus importants sont

- la consommation spécifique d'explosif :

- la régularité de la distribution de l'explosif;

- l'inclinaison des trous;

- l'utilisation des microretards:

- la construction des charges;

- la maitrise des propriétés technologiques du massif.

Les données présentées sur les tableaux et figures sont obtenues par les différentes expériences réalisées dans plusieurs carrières de matériaux de construction.

TABIEAUI Catégories de fissuration des roches.

\begin{tabular}{c|c|c|c} 
Catégorie & Roches & $\begin{array}{c}\text { Distances entre } \\
\text { les fissures }(\mathrm{m})\end{array}$ & $\begin{array}{c}\text { Densité de fissuration } \\
\text { Nombre de fissures } \\
\text { par mètre linéaire }\end{array}$ \\
\hline I & Extrémement fissurées & $<0,1$ & $>0,1$ \\
\hline II & Très fissurées & $0,1-0,5$ & $20-10$ \\
\hline III & Fissuration moyenne & $0,5-1,0$ & $1,0-2,0$ \\
\hline IV & Peu fissurées & $1,0-1,50$ & $1,0-0,65$ \\
\hline V & Monolithe, non fissurées & $>1,50$ & $<0,65$ \\
\hline
\end{tabular}

\section{Consommation spécifique d'explosif}

La pratique des travaux de tir à travers plusieurs mines à ciel ouvert montre que le changement de la consommation spécifique provoque une large variation de la fragmentation, ce qui a permis à certains chercheurs de dire que i tous les problèmes de la fragmentation sont résolus par le réglage de la consommation spécifique d'explosif $n$. Nous pensons que cette hypothèse n'est pas vraie pour toutes les roches. A cet effet, nous avons réalisé un ensemble d'essais qui ont montré que pour les roches monolithiques, l'augmentation de la charge de 2,3 fois provoque une diminution proportionnelle des classes de grosses fractions, Cette diminution n'est que de 1,3 fois pour les modules fissurés.

\section{Régularité de distribution de l'explosif}

Le diamètre du trou influe sur la concentration de l'explosif dans le massif. Ainsi I'augmentation du diamètre engendre l'augmentation de la charge concentrée et provoque le plus souvent l'augmentation du nombre de morceaux hors gabarit. Les expériences réalisées en carrières montrent que la diminution du diamètre de la charge de deux fois provoque une diminution des hors gabarit de 7 à $12 \%$ et celle de la consommation spécifique de 8 à $14 \%$. Ces mémes expériences montrent que le degré d'influence du diamètre de la charge sur la fragmentation dépend de la fissuration des roches. 
TABLEAUII Consommation spécifique d'explosif.

\begin{tabular}{c|c|c|c|c|c} 
& \multicolumn{5}{|c}{ Catégories de fissuration } \\
& I & II & III & IV & V \\
\hline $\begin{array}{c}\text { Diamètres } \\
\text { des morceaux } \\
\text { en mm }\end{array}$ & \multicolumn{5}{|c|}{ Consommation spécifiques d'explósif én $\mathrm{kg} / \mathrm{m}^{3}$} \\
\hline 300 & 1,9 & 2,0 & - & - & - \\
\hline 400 & 1,4 & 1,7 & 2,2 & - & - \\
\hline 500 & 0,8 & 1,4 & 1,6 & 2,0 & - \\
\hline 600 & 0,4 & 0,9 & 1,3 & 1,7 & 2,0 \\
\hline 700 & 0,3 & 0,6 & 1,0 & 1,4 & 1,6 \\
\hline 800 & 0,20 & 0,3 & 0,6 & 0,9 & 1,3 \\
\hline 900 & - & - & 0,4 & 0,6 & 0,9 \\
\hline 1000 & - & 0,2 & 0,2 & 0,4 & 0,7 \\
\hline 1100 & - & - & - & 0,3 & 0,4 \\
\hline 1200 & - & - & - & - & 0,3 \\
\hline & - & & & & - \\
\hline
\end{tabular}

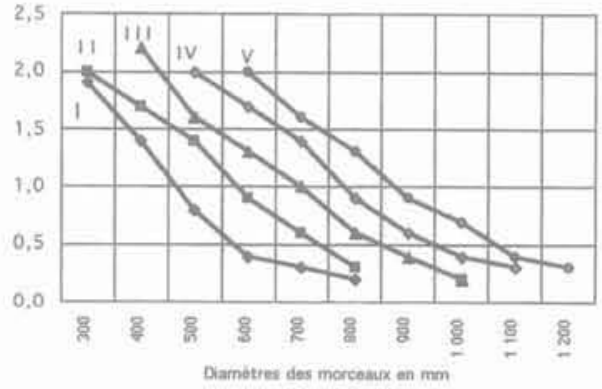

FiG. 1 La fragmentation et la consommation spécifique d'explosif dans les roches fissurées.

\section{Inclinaison des trous}

Les trous inclinés favorisent l'efficacité de la fragmentation cles roches et ce par la diminution de la zone de fragmentation réglée, les pertes d'énergie de charge cians le massif et la perte d'énergie pour la destruction du pied du gradin.

La pratique de l'abattage montre que l'utilisation de trous inclinés diminue la production des blocs hors gabarit de 8-10\% à $15-25 \%$ et augmente le rendement des pelles mécaniques de 1,5 fois (Fig. 3).

\section{Tir à microretard}

L'intervalle optimal du retard doit étre déterminé avec exactitude, puisque tout écart diminue l'effet de son utilisation. Parallèlement a la securite. I utilisation du microretard a une grande influence sur la qualité de la fragmentation. Le choix de microretard n'est pas important pour les roches dont la catégorie de fissuration est de Ià III. Par contre, pour les roches à grands blocs (catégories III à V), l'utilisation des tirs à microretard diminue la production des blocs hors gabarit de 1,6 à 2 fois.

\section{Construction des charges}

La pratique des travaux de tirs et les recherches sur ce domaine, montrent que la qualité de fragmentation et son indice

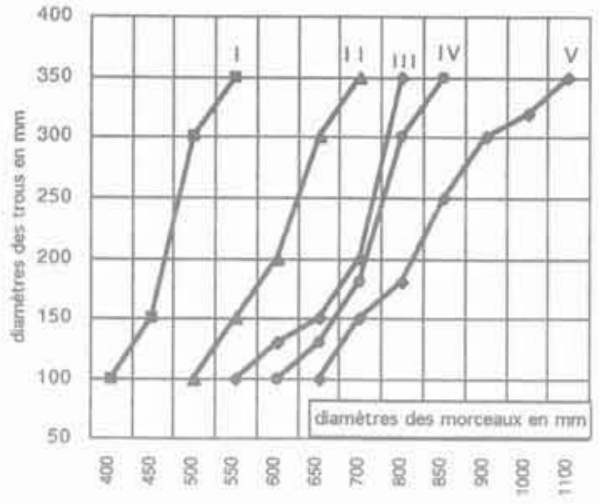

FIG. 2. Diamètres des trous et fragmentation dans les roches fissurées.

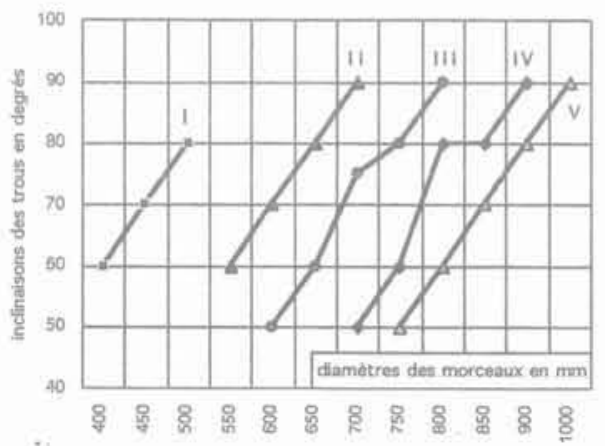

FIG. 4 Construction des charges par Y.I, Anistratov. 1 - Explosif. 2 - Bourrage. 3 Points d'amorçage. Hg - Hauteur du gradin. hp - Sous forage. hv - Espace vide. hpr-Bourrage intermédiaire.

TABLEAU III Inclinaisons des trous en degrés.

\begin{tabular}{c|c|c|c|c|c}
\hline & \multicolumn{5}{|c}{ Catégories de fissuration } \\
& I & II & III & IV & V \\
\hline $\begin{array}{c}\text { Diamètres } \\
\text { des marceaux } \\
\text { en min }\end{array}$ & \multicolumn{5}{|c}{ Inclinaisons des trous en degrés } \\
\hline 400 & 60 & - & - & - & - \\
\hline 450 & 70 & 1.4 & - & - & - \\
\hline 500 & 80 & - & - & - & - \\
\hline 550 & - & 60 & - & - & - \\
\hline 600 & - & 70 & 50 & - & - \\
\hline 650 & - & 80 & 60 & - & - \\
\hline 700 & - & 90 & 75 & 50 & - \\
\hline 750 & - & - & 80 & 60 & 50 \\
\hline 800 & - & - & 90 & 80 & 60 \\
\hline 1000 & - & - & - & 80 & 70 \\
\hline 50 & - & - & - & 90 & 80 \\
\hline
\end{tabular}



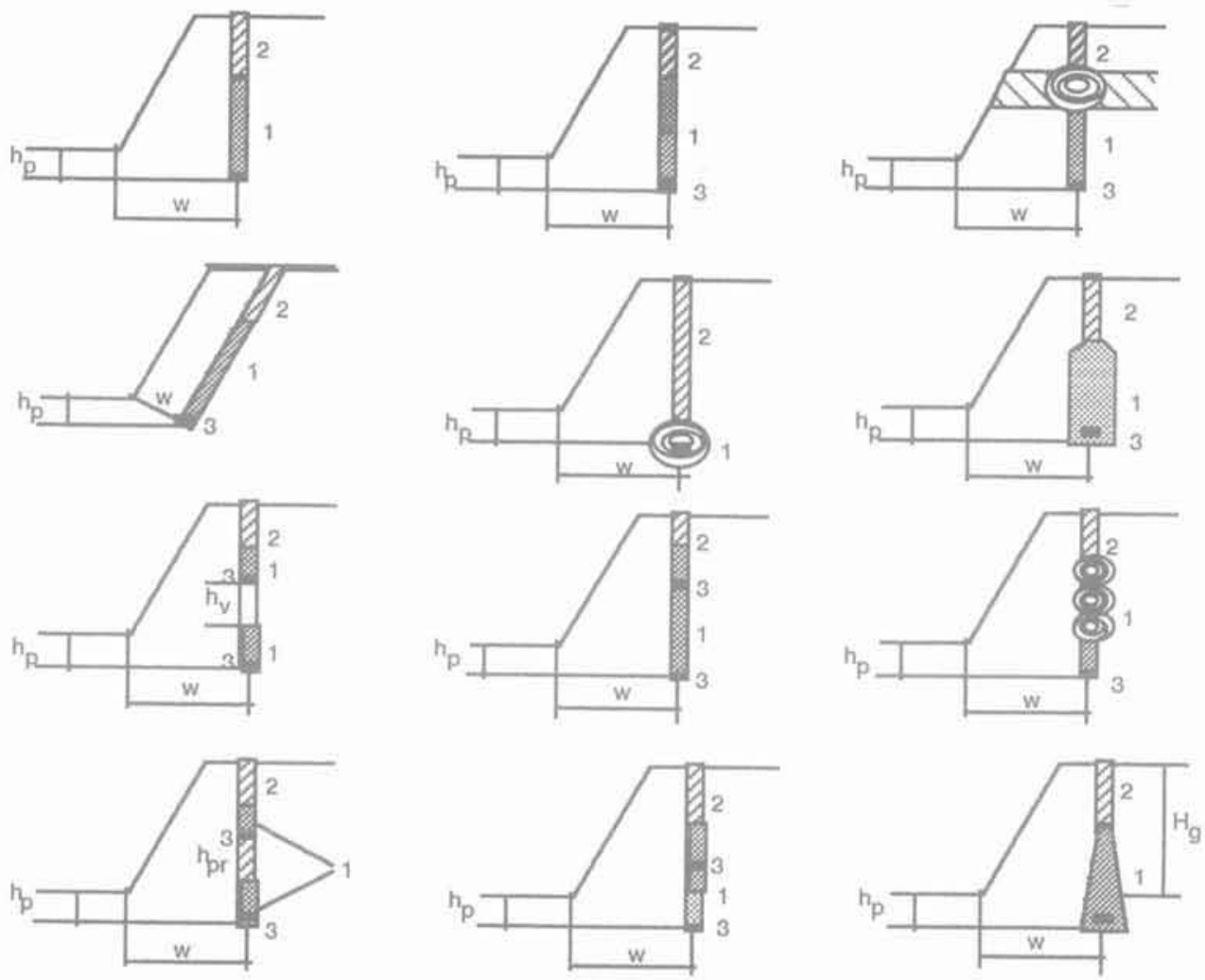

FG. 4 Construction des charges par Y.I. Anistratov, 1 - Explosif. 2 - Bourrage, 3 - Points d'amorçage. Hg Hauteur du gradin. hp - Sous forage. hv - Espace vide. hpr-Bourrage intermédiaire.

technico-économique dépendent considérablement de la construction des charges. Cette derniére est en liaison avec les propriétés des roches et avec l'exigence qualitative de la fragmentation. Toute construction de charge en carrière peut étre divisée en trois groupes selon l'indice énergétique de tir:

- les charges cylindriques correspondant aux charges verticales et inclinées continues et utilisant le même explosif:

- le deuxième groupe renferme des charges avec différents explosifs et utilisant deux ou plusieurs points d'amorçage :

- le troisième groupe renferme des charges de différentes formes et poches permettant de modifier la densité de l'explosif.
L'utilisation dés charces dispersées avec chambre d'air, améliore considérablement la fragmentation des roches. Tout comme elle permet de diminuer la consommation d'explosif jusqu'à $10 \%$ sans changement de la fragmentation. La lonqueur de l'espace d'air est généralement prise en fonction de la catégorie de fissuration des roches.

Enfin, on remarque que la qualité de la fragmentation influe directement sur le rendement des engins de chargement et de transport. Plus le diamètre moyen des morceaux est petit plus grand est le rendement de l'excavateur (pelle mécanique) et de même pour les camions.

\section{Bibliographie}

Anistratov Y. - Technologie d'exploitation des mines à ciel ouvert. Nedra, Moscou. p. 49-82, 1988.

Bastide, Deyine - Exemple de détermination d'un parc d'engins miniers à ciel ouvert. Revue de l'industrie minérale. Les techniques, janvier 1983.

Blanc J.-P. Thiar R. - L'énergie des explosifs. Explosifs, janvier 1984.

Héraud H., Mever J.-L. - Qualité de la foration, RIM. Les techniques, p. 86-98, avril 1993.
Kaufman D., Bowen R. - Analyse haul truck coasts wisely. Coal Age, CERCHAR B72 101 192, mars 1981.

Langefors U., Kihlstrom J. - The modern technique of rock blasting. Ed. Almqvist et Wiksell, Stockholm.

Martin Consultant - Surface mining equipement CERCHAR B72 108 326, 1986.

Merabet D. - Gestion de la qualité des minerais de fer dans les mines à ciel ouvert. Thèse de doctorat d'Etat, université d'Annaba, 1991.
Merabet D. - Principes de l'élaboration des projets des mines à ciel ouvert. Alger. Office des Publications Universitaires (OPU), septembre 1989.

Utler J.M. Fouts R.K. - Optimizing open pit operation with computer simulation. American mining congress journal, CERCHAR B73 80581 , march 1975 